\title{
Evaluaciones psicométricas del programa de seguimiento de niños con fenilquetonuria e hipotiroidismo congénito
}

\author{
PS. ALICIA DE LA PARRA C. ${ }^{1}$ \\ 1. Psicóloga del Programa de Seguimiento de niños PKU. Laboratorio de Enfermedades Genéticas y Metabólicas, \\ INTA - Universidad de Chile.
}

Palabras clave: Fenilquetonuria, hipotiroidismo, congénito, evaluación, pruebas, escala.

Este artículo pretende entregar información respecto a las características de las evaluaciones psicológicas que se realizan en el Programa de Seguimiento de los niños con Fenilquetonuria e Hipotiroidismo Congénito, en cuanto a periodicidad de los controles, tipo de instrumentos utilizados, ventajas y desventajas de ellos y entregar algunas observaciones a partir de la información que manejo y de la experiencia acumulada en más de doce años de participación en este programa.

\section{Introducción}

En 1992, se inicia la aplicación en Chile del Programa de Búsqueda Masiva para Fenilquetonuria (PKU) e Hipotiroidismo congénito (HC) a todos los recién nacidos. Hasta fines del año 2007 se habían diagnosticado 800 casos entre HC y PKU. Ambas patologías si no son diagnosticadas en el período de recién nacido y tratadas oportunamente provocan retraso mental severo.

Los niños una vez diagnosticados entran al Programa de Seguimiento para PKU e HC, los cuales comprenden controles con pediatra, neurólogo, nutricionista en caso de los PKU, exá- menes de laboratorio y controles psicológicos periódicos.

Los controles psicológicos tienen como objetivo evaluar el desarrollo cognitivo (psicometría) a través de la evaluación del desarrollo psicomotor y del rendimiento intelectual con el fin de comprobar que los niños se estén desarrollando normalmente. Las evaluaciones psicológicas, en última instancia, evalúan el "éxito" del programa en cuanto a prevenir el retraso mental debido a la PKU e HC.

El Ministerio de Salud realizó una revisión de las normas tanto para el Programa de Búsqueda Masiva como del Programa de Seguimiento y publicó en el año 2007 las "Normas para el óptimo desarrollo de programas de Búsqueda Masiva de Fenilquetonuria e Hipotiroidismo Congénito" ", donde se especifican los procedimiento en la toma de las muestras, las normas de laboratorio, criterios para el diagnóstico, entre otros, y el protocolo del Programa de Seguimiento de los PKU e HCT.

\section{Algo de historia}

En 1992, se formula el primer protocolo para las evaluaciones psicométrica en el Programa de Seguimiento. Se proponen como instrumento de evaluación la Escala del Desarrollo Infantil de Bayley para evaluar el desarrollo psicomotor y la Escala de Inteligencia Stanford-Binet $4^{\circ}$ Edición

Correspondencia a:

Alicia de la Parra Cieciwa

E-mail: aliciadlp@gmail.com 
para medir el rendimiento intelectual.

La primera evaluación se especifica realizarla a los 4 meses de edad, la segunda a los 6 meses y luego cada 6 meses hasta los 3 años de edad. En la edad preescolar y escolar la periocidad era cada un año a dos años en los más grandes.

Se selecciona la escala de Bayley por ser conocida internacionalmente, siendo ampliamente usada en los trabajos científicos en los que se hacían referencia a evaluaciones de lactantes e infantes. Entregaba los resultados en puntuaciones estándar de las áreas mental y motora por separado.

La escala de Stanford- Binet tenía la ventaja que con una sola prueba se podía evaluar los niños a partir de los 2 años de edad y hasta la adultez, 24 años de edad. Entregaba, además, resultados estándar por áreas específicas y un puntaje compuesto o total, con rangos similares a los entregados por las escalas de inteligencia de Wechsler, las que se utilizan habitualmente en Chile. Las áreas evaluadas por StanfordBinet son: Razonamiento verbal, razonamiento visual/abstracto, razonamiento cuantitativo y memoria a corto plazo, además del puntaje compuesto. Cada escala se expresaba en puntuaciones con un promedio de 100 y DS de 15.

En esa época se compraron algunos ejemplares de la escala intelectual y se distribuyeron en algunos hospitales públicos de la Región Metropolitana. Al poco andar, sin embargo, se descarta por no estar traducida ni adaptada ni menos había alguna estandarización hecha - o conocida- con poblaciones de habla castellana. Este hecho era especialmente limitante ya que las instrucciones de la escala señalaban que a partir de los resultados de la subprueba de vocabulario se definía el nivel de inicio de las restantes pruebas. Por otra parte, la cuarta edición de la Escala de Stanford- Binet era radicalmente distinta a la primera y no era conocida en Chile. Los psicólogos no sabían aplicarla por no haber recibido alguna capacitación para su administración y por ello no la utilizaban.

Protocolo de evaluación psicológica del Programa de Seguimiento de PKU e HC, 2007

En las normas del Programa de Búsqueda Masiva y de Seguimiento de PKU e HC se señala que las evaluaciones psicológicas comprenden:

Evaluación del desarrollo psicomotor con la Escala de Bayley a los 4, 6, 12, 18 meses de edad y luego cada un año.

Evaluación del rendimiento intelectual, se señala en las normas, se puede realizar con la Escala intelectual de Stanford-BInet o Escalas intelectual de Wechsler (WPPSI, para preescolares y WISC-R para escolares) cada uno y dos años en escolares mayores.

En el policlínico de enfermedades genéticas y metabólicas (LabGem) del INTA, Universidad de Chile, y centro de referencia para PKU en seguimiento, se ha mantenido la frecuencia cada seis meses a partir de los seis meses de edad hasta los 3 años o tres y medio años con la Escala de Bayley segunda edición. Se realizan dos evaluaciones en la edad preescolar (4 y $5 \frac{1}{2}$ años de edad aproximadamente) con WPPSI y desde los 7 años cada uno a dos años con WISC-R, usando las normas norteamericanas.

\section{Características de las pruebas}

1) Escala del Desarrollo Infantil de Bayley: En Chile, se cuenta en algunos centros y hospitales con la primera edición (1969) de Escala de Bayley y con la segunda edición (1993)². Al inicio del Programa de Seguimiento se importaron y distribuyeron algunos ejemplares en hospitales de la Región Metropolitana.

La diferencia fundamental entre ambas, es el rango de edad que mide. En la primera se evalúa niños desde el mes de vida hasta los $2 \frac{1}{2}$ años de edad y la segunda hasta los $3 \frac{1}{2}$ años de edad. Se simplificaron algunos ítemes y se eliminaron algunos por la dificultad para observar ciertas conductas o por los materiales. En la segunda versión aumenta considerablemente los ítemes verbales y se incluyen evaluaciones de áreas no vistas anteriormente con la inclusión de nuevos materiales.

Ventajas de las Escalas de Bayley, segunda edición:

- Usada internacionalmente.

- Cuenta con dos escalas mental y motora que entrega índices de desarrollo: mental (MDI) y motora (PDI). 
- Entrega resultados en puntajes estándar (promedio $=100$ y DS $=15)$ y clasificados en categoría (Desarrollo adelantado $(>115)$, Normal (85 - 115), Retraso Leve $(70-84)$ y Retraso Significativo $(<69)$.

- Estandarización fue hecha considerando diferentes poblaciones socioculturales de Estados Unidos y niveles económicos y regiones. Se evaluaron 100 niños por rango de un mes de edad (n total $=1400$ niños en la primera edición y 1700 en la segunda).

Qué mide cada escala:

Escala Mental, los ítemes de ésta escala mide entre otras las siguientes áreas:

- Lenguaje: vocalizaciones, verbalizaciones, comprensión de instrucciones, manejo de conceptos (cantidad, tamaño, género, colores, etc).

- Habilidades cognitivas como: percepción, atención, discriminación visual, memoria, integración sensorial e integración perceptualmotor; coordinación de acciones para un fin; solución de problemas, etc.

- Social: reacción ante extraño, reacción frente a imagen especular, consideración del punto de vista del otro, etc.

Escala Motora, los ítemes evalúan:

- Motricidad gruesa: tono muscular, control del cuerpo, coordinación de extremidades, etc.

- Motricidad fina: aprensión de objetos, toma de lápiz, coordinación motriz fina, etc.

Registro Conductual (resultados en percentiles y categoría: óptimo/no óptimo) evalúa 4 áreas:

- Atención/Alerta.

- Orientación / Compromiso.

- Regulación Emocional.

- Cualidad Motora.

Desventajas de las Escalas de Desarrollo Infantil de Bayley (BSID):

- A pesar de señalar que se evalúan diferentes habilidades no entrega puntuaciones por funciones o áreas. Por ejemplo, en la Escala Mental: lenguaje, razonamiento no verbal, integración intersensorial, organización perceptual, atención, responsividad social, etc. En la Escala Motora incluye tanto ítemes que miden motricidad gruesa como fina. Por lo tanto, puntuaciones bajas pueden deberse a un menor rendimiento en todas los ítemes o puede ser producto de inmadurez o dificultades en un área específica (por ejemplo, lenguaje o en motricidad gruesa solamente).

- No todas las funciones o habilidades están representadas en los distintos rangos de edad, $\mathrm{o}$ en alguno de ellos predominan algunos (por ejemplo a partir de $\operatorname{los} 2 \frac{1}{2}$ años de edad predominan ítemes de lenguaje).

- Alto número de ítemes que deben ser evaluados en cada rango (aproximadamente 25 ítemes en la Escala Mental).

- Tiempo requerido, mínimo de 30 minutos en los menores de 10 meses de edad, los mayores requieren alrededor de 40 minutos aproximadamente, sin el registro de la escala conductual.

- No ha sido estandarizada en población de habla castellana, sólo la primera edición del BSID tiene una versión traducida al español. La segunda edición está en inglés.

- Requiere tiempo para la capacitación, dado el gran número de ítemes y además es necesario la traducción. Este hecho ha causado que muchos psicólogos se han mostrado reacios a utilizar la prueba.

- Debe ser importado y es caro (aproximadamente mil dólares).

- Los materiales que se utilizan vienen en una maleta la cual es grande y pesada.

2) Pruebas de Inteligencia de Wechsler: Ventajas de las Escalas de Wechsler:

- Utilizada internacionalmente. Existen estandarizaciones en diferentes países y también en algunos de habla castellana (Argentina, España, Chile).

- Es la prueba que habitualmente se utiliza en Chile. En las escuelas de psicología se enseña y capacita en su administración y corrección.

- Existe escalas para diferentes rangos de edades: preescolar (WPPSI), escolar(WISC) y adultos (WAIS) y que mantienen, relativamente, los tipos de subpruebas y por lo tanto habilidades que se evalúan. 
- Se han actualizado cada una de las pruebas (WPPSI III, WISC - R, WISC III, WISC IV, WAIS III).

- Entrega resultados en Coeficiente Intelectual de la escala Total y de dos subescalas Verbal y Manual, con un promedio de $100 \mathrm{y}$ DS 15. Coeficiente Intelectual, es un concepto conocido y compartido por la población en general.

- La puntuación en CI están clasificadas en rangos, que en término generales son conocidos:

- Superior: CI 120 - 130 (6,7\%).

- Normal Superior: CI 110 - 119 (16,1\%).

- Normal Promedio: CI 90 - 110 (50\% de la población).

- Normal Lento: CI 80 - 89 (16,1\%).

- Limítrofe: CI 70 - 79 (6,7\%).

- Retraso o Deficiencia mental o cognitiva Leve: CI 55 - 69 (2,2\% presenta algún grado de deficiencia mental).

- Retraso Moderado: CI 40 - 54.

- Retraso Severo: CI 25 - 39.

- Las puntuaciones de las subpruebas (6 de la escala verbal y 5 de la escala manual) permiten describir un perfil de rendimiento (promedio 10 y rango normal de subpruebas 8 12) y detectar rendimiento por sobre y por bajo del rango normal o del promedio de rendimiento del sujeto.

Qué miden las pruebas de Wechsler:

- Evalúa y discrimina, razonamiento verbal y razonamiento no verbal o manual

- Escala verbal:

- Información: Nivel de información general que maneja el sujeto, lo cual requiere atención e interés en el medio y memoria a largo plazo.

- Semejanzas: Capacidad de relacionar dos conceptos verbales, abstracción verbal.

- Aritmética: razonamiento numérico, es una prueba que requiere de atención y manejo de la información en forma mental (memoria de trabajo).

- Vocabulario: conceptualización verbal.

- Comprensión: razonamiento aplicado a situaciones sociales y concretas de la vida diaria, sentido común.
- Retención de Dígitos: memoria a corto plazo de estímulos auditivos.

- Escala manual:

- Completación de figuras: atención visual, gestalt.

- Ordenación de historias: razonamiento secuencial, relaciones lógicas de situaciones sociales; sentido común, causalidad.

- Cubos: capacidad de análisis y síntesis de patrones visuales, organización y razonamiento visoespacial.

- Ensamblaje: integración de partes en un todo, capacidad de planificación y trabajo sistemático.

- Claves: aprendizaje de una tareas de asociación de estímulos visuales, rapidez visomotriz, memoria visual, concentración.

- Las versiones más actualizadas han incluido otras subpruebas que miden por ejemplo razonamiento analógico. Las puntuaciones de las subpruebas se agrupan en Índice de Comprensión Verbal, Razonamiento perceptivo, Memoria de trabajo, velocidad de procesamiento de la información ${ }^{3}$. Esto último debido a que en estudios que se evalúan funciones específicas en grupos de personas con diferentes patologías se ha visto que éste, incluyendo memoria de trabajo, atención son las más vulnerables a cualquier alteración que tenga efectos sobre el SNC.

Desventajas de las pruebas de Wechsler:

- En Chile, en general se cuenta en los servicios públicos se salud sólo con la primera versión de WPPSI y sólo con WISC-R. Ésta última que fue estandarizada en Chile entre 1974 y 1982, presenta normas que en algunos rangos de edad pareciera entregar puntajes por sobre lo que se aprecia en la observación clínica. Este hecho ha llevado a que muchos psicólogos utilicen las normas norteamericanas.

- Algunas ítemes de las subpruebas verbales están obsoletas ${ }^{2}$.

- A pesar que algunas subpruebas pudiera considerarse que tienen una carga importante de nivel cultural y educacional, éstas no son tan exigentes sino que son los que se comparten en la población general.

- Por lo general, las pruebas no son adminis- 
tradas en forma completa o no evalúan Retención de Dígitos, prueba que mide atención y memoria a corto plazo, que son unas de las funciones que generalmente se describen como afectada en PKU e HTC.

- Recientemente (2007) la Universidad Católica realizó una estandarización de WISC III pero todavía no parece estar muy difundi$\mathrm{da}^{4}$.

- Por otra parte, las pruebas que se adquieren en el país, no son las originales, por lo tanto, el material no siempre cumple con la calidad requerida.

- Algunos estudios critican que las pruebas de rendimiento intelectual presentan limitaciones para evaluar los sujetos con rendimientos muy bajos y los muy altos ${ }^{5}$.

- Las pruebas para preescolares y escolares no son totalmente equivalentes, especialmente en la escala manual ${ }^{6}$.

- Estas prueban miden RENDIMIENTO y por lo tanto, existe una variabilidad intrasujeto, al verse afectado por variables emocionales: ansiedad por el rendimiento, inseguridad, depresión.

- El tiempo necesario para administrar la escala completa y corregir es de por lo menos una hora.

\section{Características de Fenilquetonuria (PKU) e Hipotiroidismo Congénito (HTC) Fenilquetonuria (PKU)}

El tratamiento de PKU es básicamente nutricional. Consiste en la restricción estricta de proteínas y parcial de otros alimentos que contienen fenilalanina (FA), las cuales deben ser medidos y calculados según una tabla, para asegurarse de mantener niveles de FA en sangre dentro de los rangos recomendados. La FA en niveles alto es un neurotóxico y por ello provoca retraso mental severo cuando no son tratados precozmente. Los niños PKU deben además suplementarse con una leche especial que contienen aminoácidos escenciales para un buen crecimiento. Esta leche tiene sabor y consistencia que no es en todos los niños bien tolerado. Por otra parte, variables como las vacunas o las infecciones causan aumento de FA en sangre.

Estas características hacen que el control óptimo de la enfermedad sea más difícil, y por lo tanto, los PKU están más vulnerables de sufrir algún daño manifestado en deficiencias en las funciones cognitivas específicas. En los estudios se señalan que las funciones más afectadas son la atención selectiva y sostenida, desarrollo del lenguaje, visomotricidad, funciones ejecutivas, memoria de trabajo, también se observa mayor porcentaje de niños con dificultades en el aprendizaje, especialmente matemáticas.

\section{Hipotiroidismo Congénito (HTC)}

El tratamiento es más simple que PKU ya que requiere la ingesta una vez al día de Ltiroxina y por lo tanto, el control es más fácil. Los HTC diagnosticados y tratados antes de 60 días presentan un desarrollo psicomotor e intelectual normal. Sin embargo, se ha detectado que presentan en relación a controles sanos un mayor porcentaje de retraso leve del lenguaje, problemas en la atención, alteraciones mínimas en orientación visoespacial, dificultad en la coordinación visomotora, hiperquinesia. El HTC también puede provocar hipoacusia.

La incidencia de HTC es de 1:3 000 RN mayor que PKU clásica, 1:21 000, habiendo un número mayor de niños con tal enfermedad.

\section{La realidad en la aplicación del Programa de Seguimiento en PKU e HTC}

El Programa de Seguimiento de niños PKU diagnosticados en los servicios hospitalarios públicos ha logrado seguir en forma bastante aproximada el protocolo de las evaluaciones psicométricas. Esto en parte, por haberse constituido el Laboratorio de Enfermedades Genéticas y Metabólicas del INTA Universidad de Chile en lugar de referencia para ésta patología. Sin embargo, en el seguimiento de niños con HTC no ha sido tan exitoso. El seguimiento de los niños HTC de la región Metropolitana se ha centrado en el Hospital San Juan de Dios, pero los niños de otras regiones han sido controlados en los hospitales y centro de salud que les corresponden por el lugar de residencia. En una jornada que se realizó este año para evaluar el programa se vio que en muchos centros hospitalarios los profesionales que trabajan ahí no tienen claridad respecto al protocolo de con- 
troles de los HTC tanto médicos como psicológicos. Las horas psicólogos no serían suficiente como para incluir el seguimiento tal como está señalado, por la alta demanda de atención en ésta especialidad.

Además, en la mayoría de los centros no se cuenta con la Escala del Desarrollo Infantil de Bayley, en otros tampoco con la prueba para preescolares de Wechsler.

Por lo general, en los servicios públicos se utiliza para evaluar el desarrollo psicomotor desde el mes de vida hasta los dos años la Escala del Desarrollo Psicomotor (EDDP). Ésta es una prueba realizada en Chile, breve (alrededor de 6 ítemes por rango de edad), puede ser y es (o debiera ser) aplicada por otros profesionales (enfermeras como parte del control del niño sano), y entrega puntuaciones estándar en Coeficientes de Desarrollo, es una prueba fácil de adquirir y barata. De los 2 a 5 años de edad se utiliza el TEPSI, el cual también está hecho en Chile, es breve, barata, entrega resultados en tres áreas: lenguaje, coordinación y motora, pero en categorías de desarrollo normal, riesgo y retraso.

Tomando en cuenta todos los antecedentes señalados cabe preguntarse:

- ¿Debemos realizar evaluaciones psicométricas con igual periodicidad en PKU e HTC?

- ¿Debiéramos realizar evaluaciones más simples para cumplir con la periodicidad requerida pero que nos permitan detectar riesgo de retraso y realizar evaluaciones más exhaustivas en casos necesarios?

- ¿Las pruebas psicométricas son suficientes para detectar deficiencias, algunas frecuentes en estos niños, y poder referir a tratamiento especializado o a programas de estimulación en una forma oportuna?

- ¿Sería recomendable realizar a todos los niños evaluaciones más específicas en momentos críticos (por ejemplo lenguaje entre los $2 \frac{1}{2}$ a 3 años de edad, integración visomotriz a los 5 años, atención $7-8$ años)?

- ¿El Ministerio de Salud estaría dispuesto a implementar con las pruebas psicométricas mínimas a todos los centros de salud? ¿Estaría dispuesto a invertir en pruebas psicométricas actualizadas?
- ¿Sería más conveniente contar con centros, por regiones, dónde se realicen evaluaciones psicométricas específicas?

\section{En síntesis:}

La información compartida respecto a las pruebas psicométricas, el cumplimiento del protocolo de las evaluaciones psicológicas del Programa de Seguimiento, las dificultades, las observaciones y cuestionamientos planteadas, espero que hayan servido en alguna medida, en primer lugar, para los profesionales de la salud no psicólogos conozcan las pruebas psicométricas que se utilizan, qué resultados entregan, sus alcances y sus limitaciones. $\mathrm{Y}$ en segundo lugar, entregar antecedentes y aspectos que a mi parecer debieran tomarse en consideración cuando se definen los protocolos para éste o cualquier otro programa del Ministerio de Salud.

Y termino señalando y preguntando cosas tan obvias como las siguientes:

Cuán importante parece ser evaluar la factibilidad de llevar a cabo los programas propuestos, considerando las condiciones físicas, los implementos, profesionales con que se cuenta en los hospitales y centros de salud pública. Cuán importante es definir y organizar el seguimiento (¿centralizar-descentralizar?, ¿trabajo en redes?, ¿coordinar servicios?). Y un aspecto no menor, cómo motivar y comprometer a los profesionales involucrados en el cumplimiento de los protocolos. Mantenerles informados, entregar facilidades para su cumplimiento, evaluar los resultados, capacitarlos, crear instancias para compartir las experiencias y buscar a partir de ahí nuevas proposiciones y soluciones, parece ser una buena receta.

\section{Referencias}

1.- "Normas para el óptimo desrrollo de programas de búsqueda masiva de Fenilquetonuria (PKU) e Hipotiroidismo Congénito (HC) y otros errores congénitos del metabolismo." Ministerio de Salud, Santiago, Chile, 2007.

2.- Bayley N: Bayley Scales of Infant Develpment, Second Edition. San Antonio, TX: The Psychological Corporation, 1993.

3.- Escala de inteligenica de Wechsler para Niños -IV 
(WISC-IV). TEA Ediciones S.A. Madrid, 2005.

4.- Ramirez V, Rosas R: Estandarización del WISC-III en Chile: Descripción del Test, Estructura Factorial y Consistencia Interna de las Escalas. PSYKHE 2007; 16 (1): 91-109.

5.- Ripoll B: Algunos problemas de los tests de inteligen- cia en la evaluación de los alumnos/as con retraso mental: El caso del WISC-R. Revista Siglo Cero. Vol. 32 (2), no 195, 21-25. Madrid, 2001.

6.- Quereshi MY, Seitz R: Non-equivalence of WPPSI, WPPSI-R and WISC-R scores. Current Psychology 1994; 13 (3): 210-25. 